\title{
Magnetic models on Apollonian networks
}

\author{
Roberto F. S. Andrade \\ Instituto de Física, Universidade Federal da Bahia, 40210-340 Salvador, BA, Brazil \\ Hans J. Herrmann* \\ Departmento de Física, Universidade Federal do Ceará, 60450 Fortaleza, CE, Brazil
}

(Received 13 November 2004; published 31 May 2005)

\begin{abstract}
Thermodynamic and magnetic properties of Ising models defined on the triangular Apollonian network are investigated. This and other similar networks are inspired by the problem of covering a Euclidian domain with circles of maximal radii. Maps for the thermodynamic functions in two subsequent generations of the construction of the network are obtained by formulating the problem in terms of transfer matrices. Numerical iteration of this set of maps leads to very precise values for the thermodynamic properties of the model. Different choices for the coupling constants between only nearest neighbors along the lattice are taken into account. For both ferromagnetic and antiferromagnetic constants, long-range magnetic ordering is obtained. With exception of a size-dependent effective critical behavior of the correlation length, no evidence of asymptotic criticality was detected.
\end{abstract}

DOI: 10.1103/PhysRevE.71.056131

PACS number(s): 05.50. $+\mathrm{q}$, 89.75.Hc, 02.50.Cw, 64.60.Ak

\section{INTRODUCTION}

The investigation of magnetic models on scale-invariant networks has attracted the attention of scientists since the 1980s [1,2]. Besides the fact that, on such graphs, renormalization procedures can lead to exact results [3], they have been explored as models for systems that are not translationally invariant, neither in the positions of the spins nor in the coupling constants mediating the interactions between them. In this respect, the analysis of disordered and aperiodic models on scale-invariant graphs, which include hierarchical lattices [4-6], Cayley trees [7], or Sierpinski gaskets and carpets $[8,9]$, have provided valuable insight into the behavior of critical phenomena of nonhomogeneous systems on Euclidean lattices.

A further family of scale-invariant graphs are Apollonian networks (ANs), the simplest of which is illustrated in Fig. 1. This lattice can be defined based of the ancient problem of filling space with spheres, first tackled by the Greek mathematician Apollonius of Perga [10]. In its two-dimensional version, corresponding to the problem of the plane filled by circles, the nodes of this network are defined by the positions of the centers of the circles, while edges are drawn between any pair of nodes corresponding to pairs of touching circles [11]. The resulting network corresponds to the contact force network of the packing [12]. ANs can also be used to describe generically other scale-free situations, such as spacefilling porous media [13] or the connections between densely located cities, for which one is interested in fluid flow, car traffic or electric supply. Therefore, it is useful to study not only the geometric properties of ANs, but also transport and ordering on them.

In a previous paper [14], we analyzed several of the AN's properties. In particular, we have shown that it has small-

\footnotetext{
*Also at Institute for Computerphysics, University of Stuttgart, Germany. Electronic address: hans@ica1.uni-stuttgart.de
}

world properties, a scale-free degree distribution, a very high clustering coefficient, and a very short diameter, all this having been confirmed independently by Doye et al. [15]. Moreover, the AN can be embedded in the Euclidian plane, which is not the case for other scale invariant lattices, e.g., hierarchical lattices or Cayley trees. One can, of course, define other similar lattices based on modified packing rules [16]. Although the numerical values for exponents depend on the topology of each realization, basic properties characterizing complex networks remain the same.

In our first work, we devoted our attention to several physical models of the AN (electrical resistance, percolation, magnetic ordering), pointing out the most striking features. In this work, we review our investigations on the properties of several Ising models on the AN, and present a thorough discussion regarding, on one hand, some of the details of the transfer matrix (TM) methods and, on the other hand, the most important thermodynamic and magnetic properties.

The results we obtain qualitatively represent a class of models that has not yet been explored, being highly motivated by the recent development of complex networks.

We think the results discussed herein are quite relevant for the understanding of the behavior of magnetic models on lattices where interactions among spins are not restricted to their immediate geometrical neighborhood. For instance, they can be quite useful for the description of actual polydisperse packing of magnetic particles that occurs in tectonic faults since, as mentioned before, the network matches that of the contact forces. On the other hand, such lattices can be useful for modeling several other magnetic systems with disorder and long-range interaction on a microscopic level, although such models might require the inclusion of more realistic features not considered in the present study. The results for spin models can be extended to discuss properties of models for social interactions, e.g., those related to opinion formation or voter decision, where the concept of neighborhood is definitely not well described by regular lattices. 


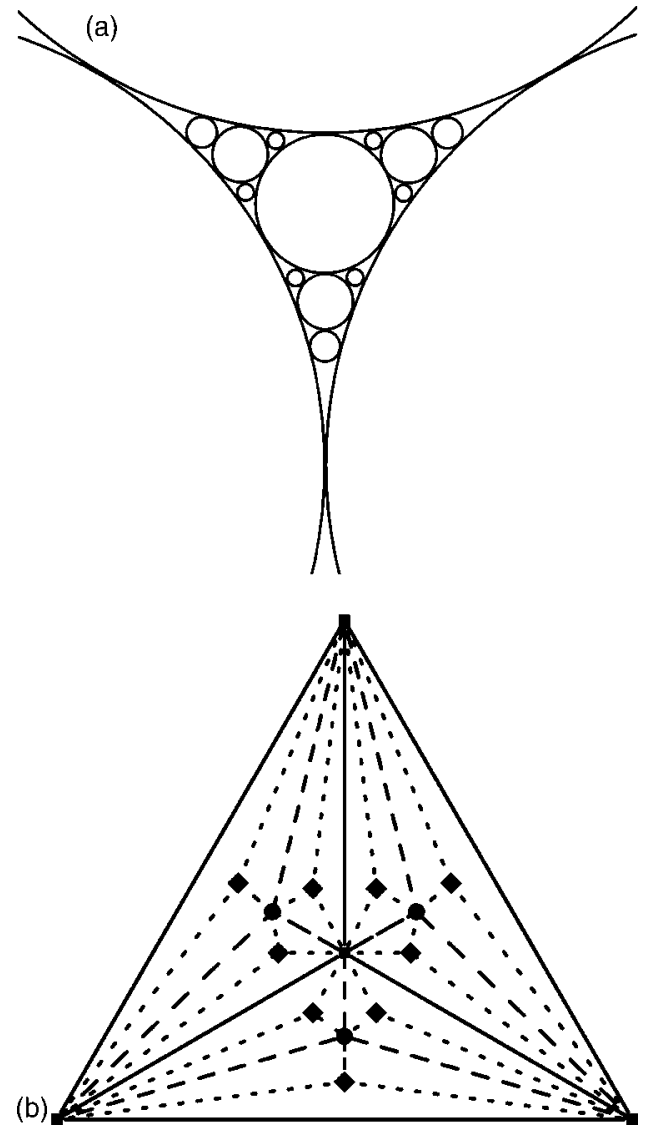

FIG. 1. Third generation $(n=3)$ of construction of the AN. In (a), we show the optimal circles that define the network. In (b), sites represented by squares, circles, and diamonds are introduced in the first, second, and third steps of construction, respectively. Links represented by dotted, dashed, and solid lines correspond to $n=3$, $m=3,2$, and 1 in Eq. (1) or $n=1,2$, and 3 in Eq. (8), respectively.

Several choices for rules defining the values of the coupling constants are considered, including both ferromagnetic (F) and antiferromagnetic (AF) interactions. As will be shown, we found long-range magnetic ordering for almost all choices of couplings without any noticeable evidence of a phase transition to a paramagnetic phase at a finite temperature. For particular choices of $\mathrm{F}$ and $\mathrm{AF}$ bonds, the geometry of the network induces the presence of competition and frustration within closed loops of an odd number of sites, giving rise to residual entropy and changes in the correlation length. The behavior of this quantity deserves a detailed discussion, as it points to a transition from long- to short-range correlation only for finite-sized systems, as discussed in a similar way for magnetic models on scale-free lattices [17].

The scale invariance in the AN topological structure can be explored in the analysis of physical models by the use of mathematical methods based on the renormalization of coupling constants or physical properties at successive stages of construction of the model. Moreover, the very precise results obtained for such models are complementary to results following from simulations for disordered models.

The rest of this paper is organized as follows: in Sec. II we discuss our models. In Sec. III we obtain the maps for the free energy and its derivatives, as well as for the correlation length. Results are discussed in Sec. IV, while concluding remarks are presented in Sec. V.

\section{ISING MODELS}

The AN is constructed recursively. In each generation, it incorporates a new set of sites, which correspond to the centers of the new circles added to the packing filling the holes left in the previous generation. In the present work we consider the lattice which starts with three touching circles drawn on the vertices of an equilateral triangle, and the packing problem is restricted to filling the space bounded by these three initial circles, as shown in Fig. 1(a). If $n$ denotes the current generation of the network, the number of sites $N(n)$ is asymptotically three times that of the previous generation; i.e., $N(n+1)=3 N(n)-5$, or $N(n)=\left(3^{n-1}+5\right) / 2$. The number of edges linking nodes increases with $n$ according to $B(n+1)=B(n)+3[N(n+1)-N(n)]$. As a consequence, $B(n)$ $=\left(3+3^{n}\right) / 2, B(n) / N(n) \rightarrow 3$ in the limit of large $n$, so that on average, each site is linked to six other sites, which is the coordination number of the triangular lattice.

Once the AN has been defined, it is possible to define many different models on it. In this work we focus on a set of interacting Ising spins $s_{i}= \pm 1$ placed on each site of the network. Interactions are restricted to pairs of spins placed on nodes linked by edges, as described in Sec. I. Thus, some spins placed far apart may interact, while pairs of spins placed relatively close to each other might not. This can be of interest to model special disordered systems that have interactions of all ranges.

For the packing problem, it is important to calculate the radius of each circle $c(i, n)$, which depends both on the generation $n$ as well as on the local environment; i.e., the generations of the three circles that it touches: $n_{p}(i, k), k$ $=1,2,3$. It is possible to include this dependence into the magnetic model, by defining coupling constants (or local fields) depending on the distance between nodes; i.e., $J\left[n, i, n_{p}(i, j)\right]$. We restrict ourselves, however, to a simpler situation, where the coupling constants $J$ only depend on the generation $n$ at which the edge was introduced into the network. In Fig. 1(b) we illustrate the first three steps of the construction of the model.

To have a physically interesting model, it may be reasonable to choose values for $J$ that increase with $n$. Indeed, when $n$ increases, the average length of the edges introduced in that generation decreases and, as the spins get closer, we might expect that the interactions among them become stronger. However, to avoid the divergence of the energy within the lattice, it is necessary to renormalize the value of all $J$ 's as $n$ increases. To accomplish this, we define $J_{n, m}, m$ $=1, \ldots, n$ as the value of the constant introduced in the $m$ th generation, when the lattice has been built up to its $n$th generation, and require that $J_{n, m}$ decreases with $n-m$. A sufficiently general choice would be

$$
J_{n, m}=\frac{( \pm 1)^{m} J_{0}}{(n-m+1)^{\alpha}},
$$

where $J_{0}$ may have ferromagnetic $(>0)$ or antiferromagnetic $(<0)$ character, the exponent $\alpha$ controls how the interactions 
decay with the difference $n-m$, and yields a possibility of choosing the interactions according to the generation at which they were introduced.

One of the extreme situations, $\alpha=0$, corresponds to equal interactions along all edges in the network. On the other hand, in the $\alpha \rightarrow \infty$ limit, the model contains only finite interactions for the subset of edges that were introduced in that last generation $n$, as illustrated in Fig. 1(b). The number of these surviving bonds is given by $B(n)-B(n-1)=3^{n-1}$, so that the average coordination number is reduced to four. Moreover, the lattice is then composed by four-sided polygons, so that competition and frustration due to the presence of antiferromagnetic bonds can never occur in this $\alpha$ limit.

To close this section, we write down the formal Hamiltonian of this model:

$$
H_{n}=-\sum_{(i, j)} \overline{J_{i, j}} s_{i} s_{j}-h \sum_{i} s_{i},
$$

where all pairs of nearest neighbors denoted by $(i, j)$ are defined according to the construction rules of the network, and the constants $\overline{J_{i, j}}$ must be chosen from the set defined in Eq. (1) according to the value of $m$ in which the edge was introduced. We also include a constant magnetic field $h$, which allows for the evaluation of magnetic properties. The notation in Eq. (2) does not include the selection of the bonds that are taken into account. The evaluation of a partition function can in fact be set up in very proper terms through the transfer matrix formalism.

\section{TRANSFER MATRIX AND RECURRENCE RELATIONS}

The numerical evaluation of the partition function for magnetic models on scale-invariant graphs with a finite number of end nodes has been performed with the help of TM derived maps for a large number of lattices and models. The problem in which we are interested in this work is also suitable to be analyzed within this framework. For the sake of simplicity, let us first consider the homogeneous case $\alpha=0$, and set $h=0$. If we consider the first generation $n=1$, we observe that a $2 \times 2 \mathrm{TM} M_{1}$, which takes into account all interactions between the sites $i$ and $k$ of Fig. 1, can be written as

$$
M_{1}=\left(\begin{array}{ll}
a_{1} & b_{1} \\
b_{1} & a_{1}
\end{array}\right)=\left(\begin{array}{cc}
a\left(a^{2}+b^{2}\right) & 2 a b^{2} \\
2 a b^{2} & a\left(a^{2}+b^{2}\right)
\end{array}\right),
$$

where $a=b^{-1}=\exp \left(\beta J_{0}\right) . M_{1}$ can be used to describe one single cell or a linear chain of triangles that are connected by their bases. It is also possible to define a $2 \times 4 \mathrm{TM} L_{1}$, that describes the interactions among sites $i, j$, and $k$, where the column labels $\kappa$ are composed from the pair $(j, k)$ according to the lexicographic order, i.e., $\kappa=2(j-1)+k$, according to

$$
L_{1}=\left(\begin{array}{llll}
c_{1} & d_{1} & d_{1} & d_{1} \\
d_{1} & d_{1} & d_{1} & c_{1}
\end{array}\right)=\left(\begin{array}{cccc}
a^{3} & a b^{2} & a b^{2} & a b^{2} \\
a b^{2} & a b^{2} & a b^{2} & a^{3}
\end{array}\right) .
$$

Of course, we note that $a_{1}=c_{1}+d_{1}$ and $b_{1}=2 d_{1}$.

Within the proposed framework, all interactions between the sites $i$ and $k$, for any higher-order generations $n$
$=2,3,4, \ldots$, should be written in terms of a single $2 \times 2$ TM's $M_{n}$, with the same distribution of matrix elements as $M_{1}$. Moreover, the matrix elements of $M_{n}$ should be written in terms of those of the matrices of the lower generation $n-1$ only. This turns out to be feasible since the Apollonian lattice, in a generation $n+1$, can be decomposed into three sublattices, each one of them being a deformed lattice of generation $n$. Since the coupling constants do not depend on the actual distance between the sites, each of the three sublattices entails the same coupling constants and magnetic structure as the $n$-lattice. Thus, a matrix $M_{n+1}$ can indeed be written in terms of three matrices $M_{n}$. To achieve this we remark that, in any generation, the three sublattices share their three outmost sites, which we label as $i, j, k$, and $\ell$. This last one occupies the geometrical center of the $(n+1)$ lattice. $M_{n+1}$ can be then defined using

$$
\left(M_{n+1}\right)_{i, k}=\sum_{j, \ell}\left(L_{n}\right)_{i, j \ell}\left(L_{n}\right)_{i, \ell k}\left(L_{n}^{t}\right)_{k, j \ell},
$$

and

$$
\left(L_{n+1}\right)_{i, j k}=\sum_{\ell}\left(L_{n}\right)_{i, j \ell}\left(L_{n}\right)_{i, \ell k}\left(L_{n}^{t}\right)_{k, j \ell} .
$$

As one can easily observe by direct evaluation of Eqs. (5) and (6), all matrices $M_{n}$ and $L_{n}$ share the same matrix element distribution as $M_{1}$ and $L_{1}$. Thus, it is possible to immediately write down recurrence relations for the elements of $L_{n+1}$ in terms of those of $L_{n}$ as

$$
\begin{gathered}
c_{n+1}=c_{n}^{3}+d_{n}^{3}, \\
d_{n+1}=c_{n} d_{n}^{2}+d_{n}^{3},
\end{gathered}
$$

from which the elements $a_{n+1}=c_{n+1}+d_{n+1}$ and $b_{n+1}=2 d_{n+1}$ can be obtained.

However, a direct evaluation of the matrix elements defined by the Eqs. (5)-(7) shows that they do not exactly describe the interactions between the sites $i$ and $k$. For instance, in the generation $n=2$, the number of magnetic bonds is equal to the number of edges $B(n=2)=6$. On the other hand, we see that the Boltzmann weights in $M_{2}$ are expressed by combinations of $\exp \left(\beta r J_{0}\right) \exp \left(-\beta s J_{0}\right)$, with $r+s=9$ instead of $r+s=6$. This is due to the fact that each one of the interactions between the site $\ell$ and its neighbors $i, j$, and $k$ appears twice in Eq. (5). To describe the thermodynamics of the system with the help of Eqs. (5)-(7), it is necessary to carry out a small correction, namely, to redefine $a$ and $b$ as $a=b^{-1}=\exp \left(\beta J_{0} / 2\right)$. With this modification, the Boltzmann weights in each element are expressed by exponentials of $\beta(r-s) J_{0}$, where $r+s=B(n)-3 / 2$. Thus, for each spin configuration, the ratio between the correct energy and that one provided by Eqs. (5) and (6) is roughly proportional to $[B(n)-3 / 2] / B(n)$, which $\rightarrow 1$ in the limit $n \rightarrow \infty$.

These definitions are sufficient for only the uniform interaction model $\alpha=0$. Further modifications in Eqs. (5)-(7) are required to obtain the correct maps for general $\alpha$. To cast this into a single recurrence relation, we first note that it is not necessary to use two labels $n$ and $m$ to insert the correct coupling constants into $M_{n}$. As these matrices are recursively 
defined, the largest and most abundant $J_{n, n}$ corresponds to the constant introduced into $M_{1}$, which is reproduced in ever growing number by the successive use of equations like (5) and (6). On the other hand, the smallest and least frequent $J_{n, 1}$ represents the constant that is inserted into the sequence of TMs exactly at the $n$th generation. Thus, we consider

$$
J_{n, m} \rightarrow \mathbf{J}_{\mathbf{n}}=\frac{( \pm 1)^{n} J_{0}}{(n-1)^{\alpha}}
$$

Note that the changes carried into the denominator of Eq. (8) requires one to explicitly set $\mathbf{J}_{\mathbf{1}}=0$, and $\mathbf{J}_{\mathbf{n}+\mathbf{1}} \rightarrow \mathbf{J}_{\mathbf{n}}$. This strategy is necessary to avoid taking into account more than once the effect of bonds introduced when $n=1$, and it is somehow equivalent to the redefinition of $a$ and $b$ discussed above.

We then modify Eqs. (5) and (6) according to

$$
\left(M_{n+1}\right)_{i, k}=\sum_{j, \ell}\left(L_{n}\right)_{i, j \ell}\left(L_{n}\right)_{i, \ell k}\left(L_{n}^{t}\right)_{k, j \ell}\left(C_{n}\right)_{i, \ell}\left(C_{n}\right)_{\ell, j}\left(C_{n}\right)_{\ell, k}
$$

and

$$
\left(L_{n+1}\right)_{i, k}=\sum_{\ell}\left(L_{n}\right)_{i, j \ell}\left(L_{n}\right)_{i, \ell k}\left(L_{n}^{t}\right)_{k, j \ell}\left(C_{n}\right)_{i, \ell}\left(C_{n}\right)_{\ell, j}\left(C_{n}\right)_{\ell, k},
$$

where the $2 \times 2$ TMs $C_{n}$ are defined by

$$
C_{n}=\left(\begin{array}{ll}
p_{n} & q_{n} \\
q_{n} & p_{n}
\end{array}\right)=\left(\begin{array}{cc}
\exp \left(\beta \mathbf{J}_{\mathbf{n}}\right) & \exp \left(-\beta \mathbf{J}_{\mathbf{n}}\right) \\
\exp \left(-\beta \mathbf{J}_{\mathbf{n}}\right) & \exp \left(\beta \mathbf{J}_{\mathbf{n}}\right)
\end{array}\right) .
$$

With these definitions, it is possible to observe that the number of nonzero coupling constants in the lattice is $B(n)-3$ so that, in the $n \rightarrow \infty$ limit, Eqs. (8)-(11) accurately describe the thermodynamic properties of the model. The recurrence maps for the matrix elements derived from (9) and (10) read

$$
\begin{gathered}
c_{n+1}=c_{n}^{3} p_{n}^{3}+d_{n}^{3} q_{n}^{3}, \\
d_{n+1}=c_{n} d_{n}^{2} p_{n}^{2} q_{n}+d_{n}^{3} p_{n} q_{n}^{2},
\end{gathered}
$$

From Eq. (7) or (11) it is possible to derive recurrence maps for the free energy $f_{n}=-T \ln \left(c_{n}+d_{n}\right) / N(n)$ and correlation length $\xi_{n}=1 / \ln \left[\left(c_{n}+d_{n}\right) /\left(c_{n}-d_{n}\right)\right]$ at two subsequent generations, $f_{n+1}=f_{n+1}\left(f_{n}, \xi_{n} ; T\right)$ and $\xi_{n+1}=\xi_{n+1}\left(f_{n}, \xi_{n} ; T\right)$ can easily be derived $[18,19]$. This set of maps can be increased by working out explicit recurrence relations for the derivatives of $f_{n}(T)$ with respect to both the temperature and the magnetic field, obtaining the entropy $s(T)$, the specific heat $c(T)$, the spontaneous magnetization $m(T)=m(T, h=0)$, and the magnetic susceptibility $\chi(T, h=0)$. For this last purpose, we have to consider $h \neq 0$ and insert it into the matrices $M_{n}$. This modification breaks the up-down symmetry of the problem, so that the matrices $M_{n}$ and $L_{n}$ have a larger number of distinct matrix elements. This is a straightforward procedure that has been carried out for other models $[18,19]$. In the Appendix we present the full set of recurrence maps used in this work.

\section{RESULTS}

We study the thermodynamic functions, i.e., the free energy $f$, the entropy $s$, the specific heat $c$, the spontaneous magnetization $m$, and the correlation length $\xi$, as a function of the temperature $T$, as shown in Figs. 2-5. They were obtained by numerically iterating the set of maps shown in the Appendix, starting with $T$ dependent initial conditions, until a value $n^{*}(T)$. This temperature dependent value is set automatically, by requiring that one (or a set) of the intensive quantities and/or the correlation length, have converged to a fixed value, within a previously established relative tolerance. This is usually $\sim 10^{-15}$, as we work with double precision variables. Convergence based only on the value of $f$ and its derivatives is much faster than for $\xi$, specially when the system is in the ordered phase. Otherwise stated, if we call $n_{x}^{*}(T), x=f, s, c, m, \xi$, the value of $n$ at which the function $x$ has converged for that particular value of $T$, then we find that $n_{\tilde{\xi}}^{*}(T)$ always assumes the largest value.

In Figs. 2(a)-2(d), we show the entropy $s$, the specific heat $c$, spontaneous magnetization $m(h=0)$, and correlation length $\xi$, for three distinct values of $\alpha$, when all coupling constants have ferromagnetic character; i.e., $J_{0}>0$ and $(+1)^{n}$ in Eq. (8). The qualitative behavior does not depend on the values of $\alpha(0,1$, and $\infty)$; i.e., whether interactions are only short $(\alpha \rightarrow \infty)$ or long range $(\alpha=0)$. For all cases we see that, for low values of $T$, long-range correlation sets in, as is evident from the spontaneous magnetization and the numerical divergence of $\xi$. The remarkable feature, however, points to the absence of any criticality when $T$ is increased. The insert in Fig. 2(b) shows that, when $\alpha=0, m$ goes to zero smoothly, as $\exp (-T)$, with no evidence of a sharp transition to $m=0$ at a well-defined critical temperature. If we consider $\alpha>0$, we still find a smooth, but stronger, decay; namely, as $m \sim \exp \left(-T^{\lambda}\right)$. The curve for the specific heat is also smooth, showing a typical Schottky maximum, again without any evidence of a divergence, that would be expected for a usual phase transition.

The results for the correlation length $(d)$ are also distinct from those found for other scale-invariant models, as the diamond hierarchical lattice [19]. There, $\xi$ is finite for large values $T$ and numerically diverges for all values of $T$ below a well-defined critical value $T_{c}$ which in our case means attaining a value larger than $10^{16}$, the largest allowed number in our algorithm. Within this region, the actual value reached by $\xi(T)$, has no precise meaning. Typically it is much higher than those in the disordered phase, and is also characterized by the presence of random fluctuations. As mentioned before, $n_{\xi}^{*}(T)$ is larger than $n_{x}^{*}(T), x=f, s, c, m$, but even if we stop the iterations at $n_{f}^{*}(T), \xi$ has already reached this very high plateau. This shows that it is not actually necessary to proceed further with the iteration of the maps, as we would obtain only a meaningless value for $\xi$.

In the present case, if we use $n_{f}^{*}(T)$ to stop the iteration of the maps, we observe that $\xi$ diverges at low temperatures, expressing long-range order. When $T$ is increased beyond a given value of $T^{*}$, it converges to a well-defined value, suggesting the break of long-range correlation. However, if the iteration procedure is pursued to a value of $n>n_{f}^{*}(T)$ we 

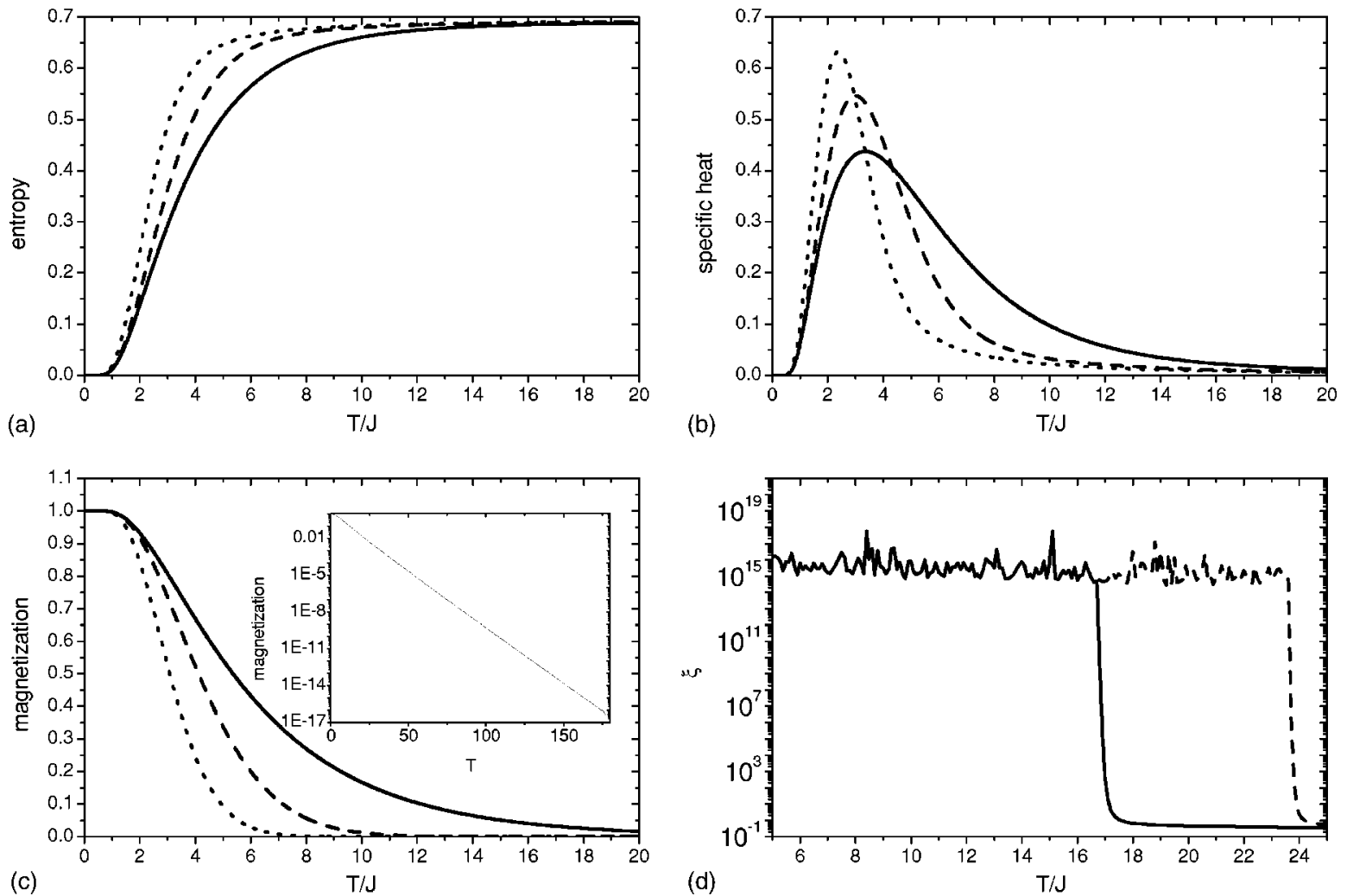

FIG. 2. Thermodynamic functions for the ferromagnetic model. Solid, dashed, and dotted lines indicate $\alpha=0,1$, and $\infty$, respectively.

observe that the $T$ interval in which $\xi$ diverges becomes larger. This finding has driven us to proceed with the iteration of the maps in a different way. We fix a value $\bar{n}>n_{f}^{*}(T=1)$, and iterate the maps until reaching $\bar{n}$ for all values within a large $T$ interval, as shown in Fig. 2(c). It is then possible to precisely evaluate a critical value $T_{c}(\bar{n})$, as the value of $T$ where the behavior of $\xi$ changes. In Fig. 3 we show how $T_{c}(\bar{n})$ depends on $\bar{n}$, for several distinct values of $\alpha$. Our findings for this unusual kind of critical behavior suggest a power law $T_{c}(n) \sim n^{\tau(\alpha)}$, with $\tau$ going continuously from $\tau(\alpha=0)=1$ to $\tau(\alpha=\infty)=1 / 2$. We recall that a similar

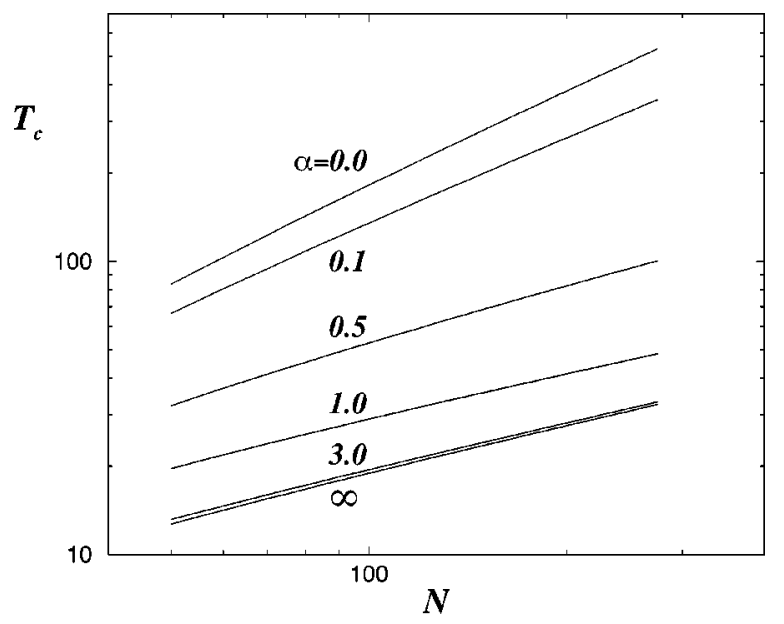

FIG. 3. Size-dependent critical temperature for distinct values of $\alpha$. behavior has been reported for spin models on another scalefree lattice [17].

In Fig. 4 we show that antiferromagnetic interactions $\left(J_{0}<0\right)$ change the thermodynamic behavior of the model. The most interesting situation is observed for $\alpha=0$. All triangles in the lattice are frustrated and, as expected, a residual entropy $s_{0}=0.222$ is measured. This best numerical value is smaller than that for the triangular lattice $s_{0}=0.3238 \ldots$ [20], and much smaller than that obtained for the Ising model on the Sierpiski gasket $s_{0}=0.493 \ldots$ (SG) [21-23].

At the same time, we find finite well-defined values for $\xi$ for all values of $T$, which are robust with respect to the value of $\bar{n}$ where the iterations are stopped. This is illustrated in Fig. 4(d), which also shows that, as $T \rightarrow 0, \xi$ decays like $\exp (-1 / T)$, typical for the one-dimensional chain. This is a somewhat unexpected behavior, as the presence of frustration usually does not allow for long-range correlation of spin orientation, even at $T=0$ (e.g., the AF Ising model on the SG [23]). At the same time, this result must be related to the nonvanishing behavior for the magnetization, shown in Fig. 4(c). It indicates that the number of spins pointing in each direction is not the same. Once again, this behavior is different from that obtained for other frustrated lattices, like the planar triangular lattice or the SG. Finally, the behavior of the specific heat looks like those found when $J_{0}>0$, for any value of $\alpha$.

In Fig. 4 we also draw curves for the thermodynamic functions when $\alpha=1$, and $\infty$. In Fig. 4(c) we see that the magnetization curve always saturates at $m(T \rightarrow 0, h \rightarrow 0)$ $=1 / 3$. This indicates that, in this limit, the number of spins pointing in opposite directions is not the same, as is the case 

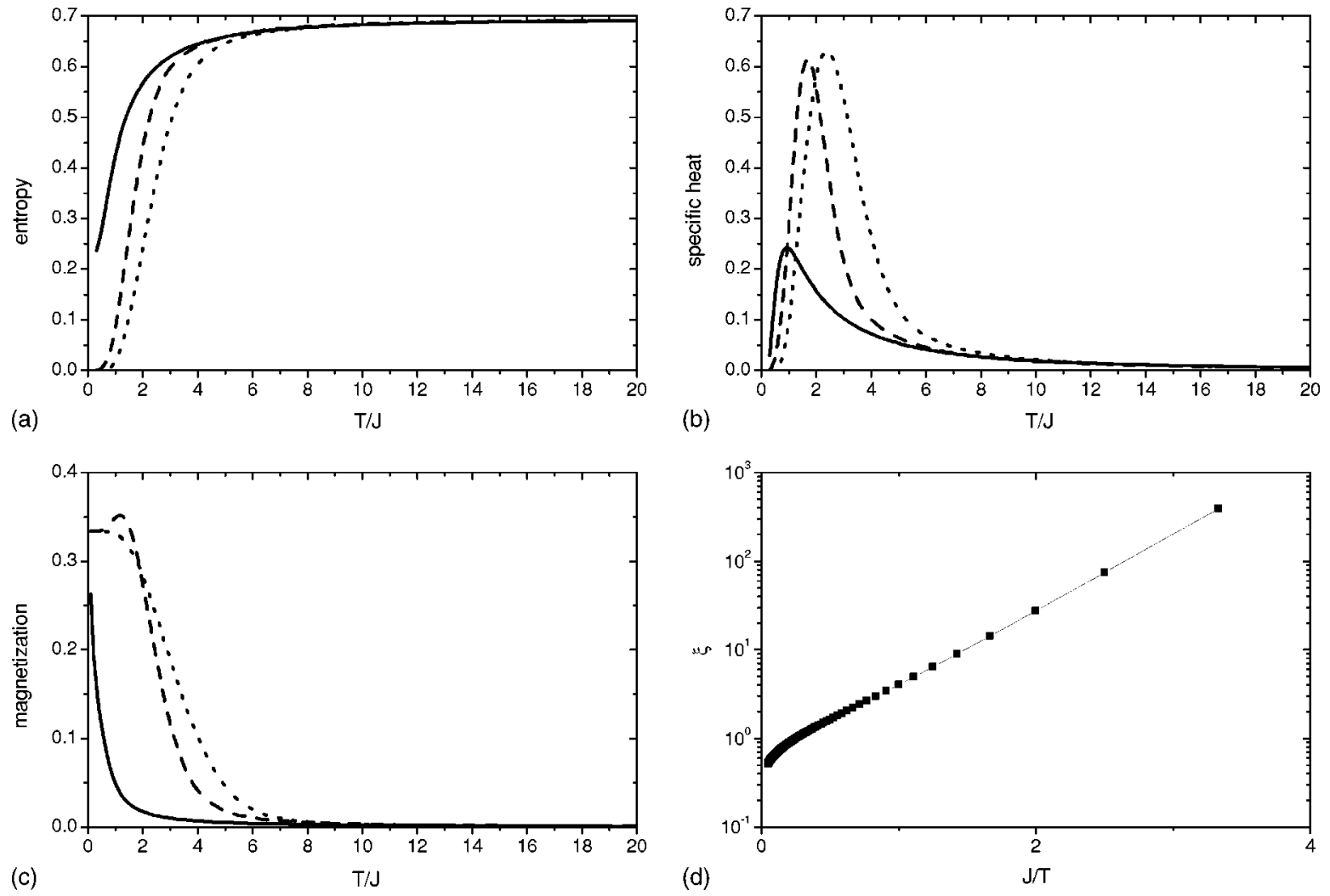

FIG. 4. Thermodynamic functions for the antiferromagnetic model. Solid, dashed, and dotted lines indicate $\alpha=0$, 1, and $\infty$, respectively. $\xi$ is drawn only for $\alpha=0$ and reciprocal temperature axis.

for the triangular lattice, but stay in proportion $2 / 3$ to $1 / 3$ independent of $\alpha$. For some range of values of $\alpha, m$ goes through a maximum [e.g., at $(T, m)=(1.2,0.351)]$ when $\alpha$ $=1$, so that a reentrant behavior at low temperatures is observed.

Let us also discuss how the presence of interactions with different signs affects the behavior of the system; i.e., when we take $(-1)^{n}$ in Eq. (8). As expected, the result depends on whether $J_{0}>0$ or $<0$. In the first case, competition and frustration give rise to residual entropy when $\alpha=0$, as illustrated in Fig. 5(a). However, we note that the value of $s_{0}$ is smaller $(\sim 0.152)$ than in the case of equal $\mathrm{AF}$ interactions. This happens because not all triangular units are built by an odd number of AF bonds, as we can easily see by inspecting the first generations with the help of Fig. 1(b). For $\alpha=0$, we note as well the presence of a double Schottky peak in the specific heat [Fig. 5(b)]. For $\alpha \neq 0$ there is no remarkable difference between the curves for $s$ or $c$ with respect to those obtained for interactions with the same sign. The results also show that the low-temperature magnetization saturates at the value $m=7 / 9$ only when $\alpha=0$; otherwise $m=1$.

Finally, when $J_{0}<0$ and alternating sign are considered, no frustrated bonds and, consequently, no residual entropy is found. The curves for the specific heat are also smooth like all other cases. The magnetization curves saturate again to $m=1 / 3$ as $T \rightarrow 0$ for all values of $\alpha$. However, reentrant behavior similar to that found for some of the AF cases has not been observed, so that the typical shape is that shown in Fig. $5(\mathrm{c})$.

\section{CONCLUSIONS}

We have studied a family of Ising models on an AN using the transfer matrix technique. On one hand we considered ferro-magnetic and antiferromagnetic couplings and, on the other hand, we generalized the interaction as being dependent on the generation, in the sign as well as in the strength quantified by an additional parameter $\alpha$.

For purely ferromagnetic couplings, we always find order in the thermodynamic limit independent of $\alpha$, which is in agreement with what has been found on other scale-free lattices [17]. Interestingly, the effective critical temperature at which the correlation length diverges goes to infinity with the system size with a power law in the number of generations with an exponent that depends on $\alpha$. For antiferromagnetic couplings we find a disordered phase for any finite temperature, but a diverging correlation length at $T=0$. This latter observation is unusual as it does not appear, for instance, on the Sierpinski gasket [23].

Considering the AN as a model for the connections between cities as described in Ref. [14] our result can be applied to the formation of opinions wherein spin up means one opinion and spin down the other one. The results for the ferromagnetic case implies that independent of the strength of the couplings between the cities as long as it is not zero, one single opinion will finally prevail.

If the Appolonian network describes the force lines in a dense polydisperse packing with each particle having a magnetic moment, as is the case in tectonic faults [24], our result for the ferromagnetic Ising model would imply that if all 

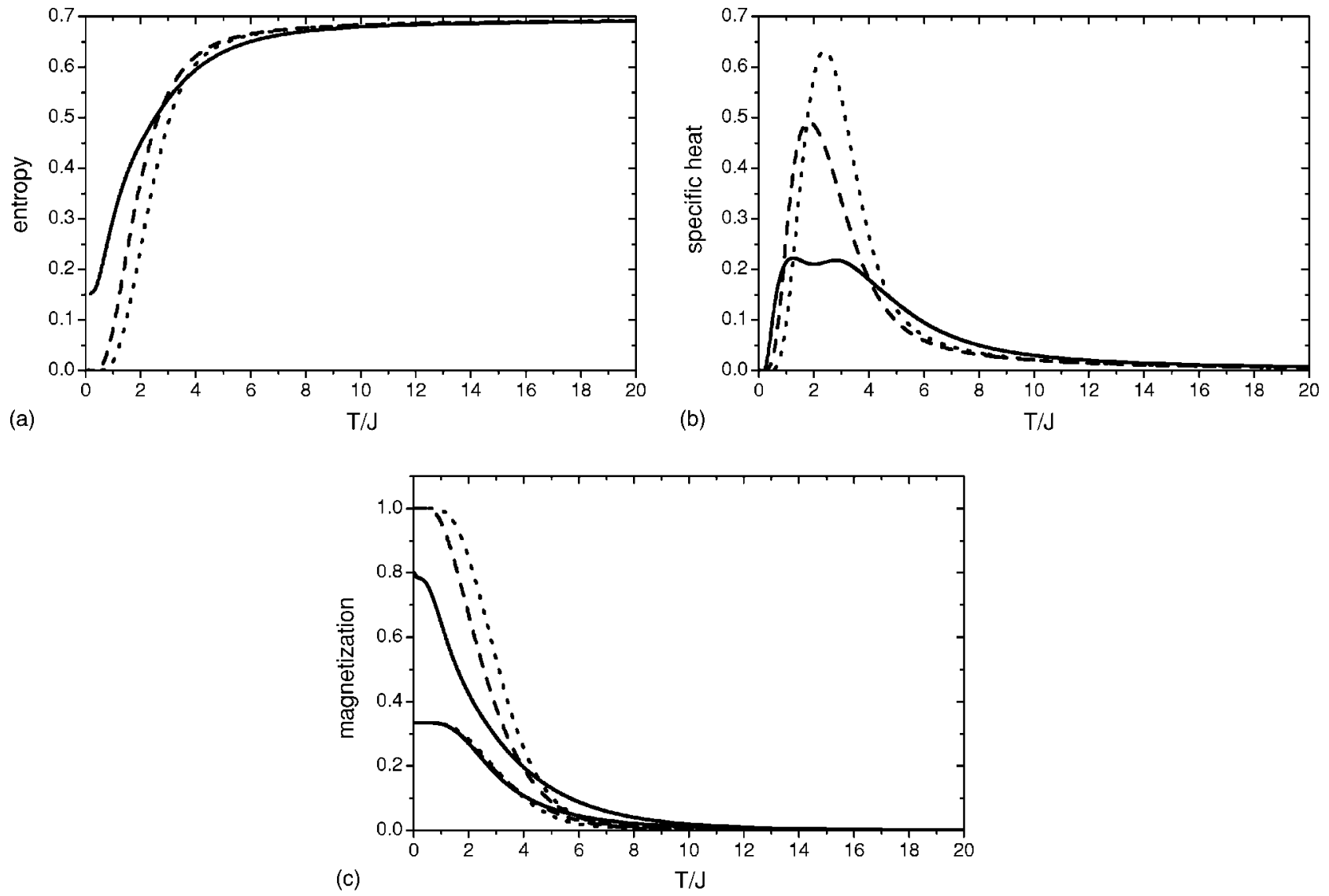

FIG. 5. Thermodynamic functions for the model with alternating ferro- and antiferromagnetic coupling, starting with $J_{0}=1$. Solid, dashed, and dotted lines indicate $\alpha=0,1$, and $\infty$ respectively, and $J_{0}>0$. In (c), three curves for the magnetization when $J_{0}=-1$ and same values of $\alpha$ saturate at $m=1 / 3$ are also drawn.

particles have a moment of equal strength, one always finds a spontaneous magnetization.

Our calculations can be generalized to random couplings (spin glass) which, in fact, is work in preparation. One can also imagine studying other more complex magnetic models on the ANs, such the Potts model, the XY model, or the Heisenberg model, and one can also study the magnetic properties of Apollonian packings of different topologies or higher dimensions, and even the case of the random Apollonian packing [25].

\section{ACKNOWLEDGMENTS}

H. J. Herrmann thanks the Max Planck Research for support R. F. S. Andrade was partially supported by CNPq.

\section{APPENDIX}

The maps for the free energy and correlation length derived from Eqs. (12) read

$$
\begin{aligned}
f_{n+1}= & \frac{3 N_{n} f_{n}}{N_{n+1}}-\frac{T}{N_{n+1}}\left\{3 \ln \alpha_{n}+\ln \left\{1+3 z_{n} \beta_{n}\left(2+\beta_{n}\right)\right.\right. \\
& +3 z_{n}^{2}\left[1+2 \beta_{n}\left(1+\beta_{n}\right)^{2}\right] \\
& \left.\left.+z_{n}^{3}\left(1+2 \beta_{n}\right)\left(2+\beta_{n}\right)^{2}\right\}-6 \ln 2\right\} \\
\xi_{n+1}= & \xi_{n}\left[1+\xi_{n}\left(\operatorname { l n } \left\{1+3 z_{n} \beta_{n}\left(2+\beta_{n}\right)+3 z_{n}^{2}\left[1+2 \beta_{n}\left(1+\beta_{n}\right)^{2}\right]\right.\right.\right. \\
+ & \left.z_{n}^{3}\left(1+2 \beta_{n}\right)\left(2+\beta_{n}\right)^{2}\right\} \\
- & \ln \left[z_{n}^{-1} \beta_{n}^{4}+z_{n}\left(1+4 \beta_{n}+2 \beta_{n}^{2}+2 \beta_{n}^{3}\right)\right. \\
+ & z_{n}^{2}\left(4+10 \beta_{n}+11 \beta_{n}^{2}+2 \beta_{n}^{3}\right) \\
+ & \left.\left.\left.z_{n}^{2}\left(3+10 \beta_{n}+10 \beta_{n}^{2}+4 \beta_{n}^{3}\right)\right]\right)\right]^{-1}
\end{aligned}
$$

where $z_{n}=\left(c_{n}-d_{n}\right) /\left(c_{n}+d_{n}\right), \quad \alpha_{n}=p_{n}+q_{n}, \quad \beta_{n}=\left(p_{n}-q_{n}\right) /\left(p_{n}\right.$ $\left.+q_{n}\right)$, and $N_{n}=N(n)$. 
[1] Y. Gefen, B. B. Mandelbrot, and A. Aharony, Phys. Rev. Lett. 45, 855 (1980).

[2] Y. Gefen, A. Aharony, Y. Shapir, and B. B. Mandelbrot, J. Phys. A 17, 435 (1984).

[3] A. A. Migdal, Zh. Eksp. Teor. Fiz. 69, 1457 (1975).

[4] A. N. Berker and S. Ostlund, J. Phys. C 12, 4961 (1979).

[5] M. Kaufman and R. B. Griffiths, Phys. Rev. B 24, R496 (1981).

[6] C. Tsallis and A. Magalhães, Phys. Rep. 268, 305 (1996).

[7] C. S. O Yokoi, M. J. de Oliveira, and S. R. Salinas, Phys. Rev. Lett. 54, 163 (1985).

[8] J. H. Luscombe and R. C. Desai, Phys. Rev. B 32, 1614 (1985).

[9] B. Bonnier, Y. Leroyer, and C. Meyers, Phys. Rev. B 37, 5205 (1988).

[10] D. W. Boyd, Can. J. Phys. 25, 303 (1973).

[11] H. J. Herrmann, G. Mantica, and D. Bessis, Phys. Rev. Lett. 65, 3223 (1990).

[12] R. Mahmoodi Baram, H. J. Herrmann, and N. Rivier, Phys. Rev. Lett. 92, 044301 (2004).
[13] J. A. Dodds, J. Colloid Interface Sci. 77, 317 (1980).

[14] J. S. Andrade Jr., H. J. Herrmann, R. F. S. Andrade, and L. R. Silva, Phys. Rev. Lett. 94, 018702 (2005).

[15] J. P. K. Doye and C. P. Massen, Phys. Rev. E 71, 016128 (2005).

[16] G. Oron and H. J. Herrmann, J. Phys. A 33, 1417 (2000).

[17] A. Aleksiejuka, J. A. Holyst, and D. Stauffer, Physica A 310, 260 (2002).

[18] M. Kohmoto, L. P. Kadanoff, and C. Tang, Phys. Rev. Lett. 50, 1870 (1983).

[19] R. F. S. Andrade, Phys. Rev. E 59, 150 (1999).

[20] G. H. Wannier, Phys. Rev. 79, 357 (1950).

[21] M. P. Grillon and F. G. B. Moreira, Phys. Lett. A 142, 22 (1989).

[22] R. B. Stinchcombe, Phys. Rev. B 41, 2510 (1990).

[23] R. F. S. Andrade, Phys. Rev. B 48, 16095 (1993).

[24] S. Roux, A. Hansen, H. J. Herrmann, and J.-P. Vilotte, Geophys. Res. Lett. 20, 1499 (1993).

[25] T. Zhou, G. Yan, P.-L. Zhou, Z.-Q. Fu, and B.-H. Wang, e-print cond-mat/0409414. 